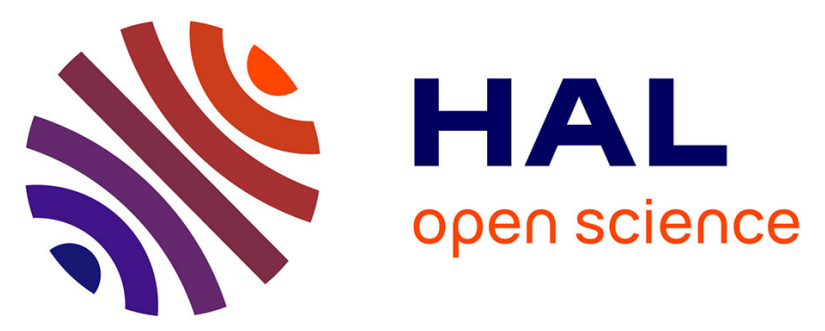

\title{
The effect of word prediction settings (frequency of use) on text input speed in persons with cervical spinal cord injury: a prospective study
}

Samuel Pouplin, Nicolas Roche, Jean-Yves Antoine, Isabelle Vaugier, Sandra

Pottier, Marjorie Figere, Djamel Bensmail

\section{To cite this version:}

Samuel Pouplin, Nicolas Roche, Jean-Yves Antoine, Isabelle Vaugier, Sandra Pottier, et al.. The effect of word prediction settings (frequency of use) on text input speed in persons with cervical spinal cord injury: a prospective study. Disability and Rehabilitation, 2016, 38, 10.1080/09638288.2016.1193229 . hal-01344978

\section{HAL Id: hal-01344978 \\ https://hal.science/hal-01344978}

Submitted on 19 Aug 2016

HAL is a multi-disciplinary open access archive for the deposit and dissemination of scientific research documents, whether they are published or not. The documents may come from teaching and research institutions in France or abroad, or from public or private research centers.
L'archive ouverte pluridisciplinaire HAL, est destinée au dépôt et à la diffusion de documents scientifiques de niveau recherche, publiés ou non, émanant des établissements d'enseignement et de recherche français ou étrangers, des laboratoires publics ou privés. 
TITLE: The effect of word prediction settings (frequency of use) on text input speed in persons with cervical spinal cord injury: a prospective study

RUNNING HEAD: The effect of word prediction settings on text input speed.

\section{AUTHORS}

Samuel POUPLIN ${ }^{1-4}$, Nicolas ROCHE ${ }^{3-5}$, Jean-Yves ANTOINE ${ }^{6}$, Isabelle VAUGIER ${ }^{4}$, Sandra POTTIER ${ }^{4}$, Marjorie FIGERE ${ }^{4}$, Djamel BENSMAIL ${ }^{1-4}$

${ }^{1}$ New Technologies Plate-Form, AP-HP, Raymond Poincaré Teaching Hospital, Garches, France.

${ }^{2}$ Physical Medicine and Rehabilitation Department, AP-HP, Raymond Poincaré Teaching Hospital, Garches, France.

${ }^{3}$ Inserm Unit 1179, Team 3: Technologies and Innovative Therapies Applied to Neuromuscular diseases, University of Versailles St- Quentin-en-Yvelines, France.

${ }^{4}$ Clinical Innovations Center 1429, AP-HP, Raymond Poincaré Teaching Hospital, Garches, France.

${ }^{5}$ Physiology-Functional Testing Ward, AP-HP, Raymond Poincaré Teaching Hospital, Garches, France

${ }^{6}$ University of François Rabelais of Tours, Tours, France 


\section{Corresponding author:}

\section{Samuel POUPLIN}

Plate-Forme Nouvelles Technologies

Hôpital Raymond Poincaré

104 boulevard Raymond Poincaré

92380 Garches, France

Tel : (33) 0147107061

Email : samuel.pouplin@aphp.fr

pouplin.samuel@gmail.com

\section{Key words:}

Cervical spinal cord injury, text input speed, word prediction software, frequency of use, computer 


\section{Abstract \\ Purpose}

To determine whether activation of the frequency of use and automatic learning parameters of word prediction software has an impact on text input speed.

\section{Methods}

Forty-five participants with cervical spinal cord injury between C4 and C8 Asia A or B accepted to participate to this study. Participants were separated in two groups : a high lesion group for participants with lesion level is at or above C5 Asia AIS A or B and a low lesion group for participants with lesion is between C6 and C8 Asia AIS A or B. A single evaluation session was carried out for each participant. Text input speed was evaluated during 3 copying tasks:

-without word prediction software (WITHOUT condition)

-with automatic learning of words and frequency of use deactivated (NOT_ACTIV condition) -with automatic learning of words and frequency of use activated (ACTIV condition) Results

Text input speed was significantly higher in the WITHOUT than the NOT_ACTIV $(p<0.001)$ or ACTIV conditions ( $p=0.02$ ) for participants with low lesions. Text input speed was significantly higher in the ACTIV than in the NOT_ACTIV $(p=0.002)$ or WITHOUT $(p<0.001)$ conditions for participants with high lesions.

\section{Conclusions}

Use of word prediction software with activation of frequency of use and automatic learning increased text input speed in participants with high-level tetraplegia. For participants with low-level tetraplegia, use of word prediction software with frequency of use and automatic learning activated only decreased the number of errors. 


\section{Introduction}

In daily modern life, Information and Communication Technologies are important for social and professional integration [1]. However, access to technology can be difficult for persons with disabilities such as cervical spinal cord injury (SCI). Different devices have been developed to enable them to use a computer depending on the level of lesion (infrared camera, trackball, onscreen keyboard, mouthsticks, hand splints) [2] [3] [4] [5] [6] [7]. Despite the development of these different assistive technologies, text input speed (TIS) remains lower for people with sensory motor impairments than for able-bodied people [8]. Several methods have been developed to increase TIS [7] [9] [10] [11] [12] [13] such as speech recognition systems [14] and word prediction software (WPS). These software, recommended by health-related professionals, are complementary: for example, in a noisy home environment, the use of a speech recognition system may be compromised. WPS may be a more appropriate solution to compensate for some of the disadvantages of speech recognition software. WPS involves displaying a list of words that relate to the word being typed by the user. If one of the predicted words is correct, the user can select it in the list, thereby avoiding having to type the whole word (keystroke saving). The prediction is based on the first letters of the word being typed (lexicon checking). However, advanced WPS also take into account the previous word within the sentence (syntactic or even semantic prediction [15]). Such WPS can be customized using different settings[16]. Among them, it is frequently possible to activate the automatic learning of new words, and to order the prediction list according to the frequency of occurrence of words in the language: the most frequently used words are thereby displayed first in the prediction list (frequency of use). The effect of some of these parameters has been studied in the literature [17], however the effect of the majority of parameters has not been evaluated. WPS are based on the frequency of words in English or 
French language. Some WPS cannot be adapted to the user's vocabulary and the frequency of words used if used in French, however other WPS allow this parameter to be activated or deactivated. To our knowledge, there are no studies in the literature which have evaluated the influence of this parameter on TIS. According to our clinical experience in persons with cervical SCI Asia A and B, it seems logical to hypothesize that the use of word prediction software and the adaptation to the user's vocabulary should allow the selection of the most appropriate words most rapidly, therefore improving TIS. Similarly, it is possible that these parameters may not have the same effect in persons with high (injury level is at or above C5

Asia A and B) and low (injury level is at or below C6 Asia A and B) tetraplegia since they do not use the same devices to access the computer.

The aim of this study was therefore to determine whether the use of WPS with the frequency of use and automatic learning parameters activated had an impact on TIS and if there was a difference between the effects in persons with high and low tetraplegia. The main hypothesis was that the activation of these parameters should improve TIS in a sample of persons with cervical SCI AIS A or B who had important motor alterations.

\section{Materials and Methods}

\section{Participants}

This study was carried out between October 2013 and March 2014. During this period, persons with cervical SCI followed in the department of physical medicine and rehabilitation of a Teaching Hospital were included if they were over 18 years old, had a SCI level between C4 and C8 Asia AIS A or B, were computer users but did not use the WPS function (to limit bias) and could read and write French. Participants were excluded if they had cognitive, linguistic or visual impairments. The study was approved by the local ethics committee (CPP 
Ile de France, Saint Germain en Laye) and all subjects provided written informed consent before participation. Data collection was finalized in April 2014.

\section{Materials.}

In order to standardize the conditions of the evaluation, a Dell XPS computer and a Toshiba computer, both equipped with a KeyVit Onscreen Keyboard and Skippy WPS were used (Skippy active state was only on the Toshiba computer, never on the Dell). Both computers have the same screen size, screen resolution, brightness and the same operating system. The WPS was chosen following a study of the most commonly prescribed or used WPS performed by our group [16] in health related professionals and persons with cervical SCI. The results showed that Skippy (a syntax-based WPS) was the most commonly used software.

Participants who used an onscreen keyboard used their usual head-controlled pointing devices.

WPS was configured to display a list of 6 words horizontally at the top of the screen. These choices were based on a preliminary study conducted in our group on the use of WPS [16] and data in the literature. Two computers were used. On a Dell computer, two parameters of Skippy were not activated: the automatic learning of new words and the faster presentation of the words most frequently used (frequency of use). The predicted words were displayed alphabetically. On a Toshiba Computer, the automatic learning of new words and frequency of use parameters were activated. Furthermore, the words of the texts used for the assessment were integrated into the WPS dictionary.

\section{Study design.}

Firstly, the WPS was explained to each participant. Then, each participant carried out a 5minute training session using WPS with the automatic learning of words and frequency of use 
parameters activated during a copying task. Finally, each participant underwent a single evaluation session. During this evaluation, three 10-minute copying tasks were carried out in a randomised order:

-a copying task without WPS (WITHOUT condition) (Dell computer)

-a copying task using WPS but with automatic learning of words and frequency of use not activated (NOT_ACTIV condition) (Dell computer)

-a copying task using WPS with automatic learning of words and frequency of use activated (ACTIV condition) (Toshiba computer)

A five-minute break was given between each copying task. Four 500-words texts of similar complexity were used, drawn from a speech and language therapy book [18]. The average word length was $5.1 \pm 0.5$ (SD) characters. The length of all the texts was deliberately too long to allow the task to be completed in 10 minutes. The evaluation was stopped after 10 minutes, no matter how much of the text had been copied. The texts were randomly allocated in order to ensure that the same text was not associated with the same condition.

Participants were instructed to use the WPS as desired; i.e., no instructions were given regarding strategy of use. Errors could be corrected.

All assessments were performed by the same investigator and were videotaped. The videos were used for the analysis.

\section{Outcome Measures.}

During the 3 copying tasks, TIS was evaluated quantitatively and qualitatively as follows: 
Quantitative assessment. Characters per minute (cpm): The number of characters typed in ten minutes divided by 10 , including punctuation marks and spaces as well as backspace, selection errors, and correction times.

- Item selection speed (items per minute): The number of items selected in ten minutes divided by 10 including punctuation marks, spaces, backspaces, arrow keys and words selected in the word prediction list.

- Number of errors and rate of word prediction use in ten minutes were noted and were calculated from videos (number of errors and number of words selected from the word prediction list in ten minutes).

Qualitative assessment (self-evaluations). Fatigue was evaluated using a 0-10 point visual analogue scale (VAS) before and after each task (0: no fatigue / 10: exhaustion)

Perception of speed and cognitive load were also evaluated using 0-10 point VASs. Perception of speed - 0: very slow / 10: very fast; cognitive load - 0: low cognitive load / 10: high cognitive load.

Satisfaction of task completion was evaluated using a 0-5 point VAS (0: not satisfied / 5: very satisfied)

\section{Data Analysis}

Descriptive statistics (mean \pm standard deviation) were used to describe continuous variables and frequencies for categorical variables.

The data followed a normal distribution (Shapiro-Wilk-test) thus parametric tests were used. In order to evaluate interactions between the effect of "frequency of use" and "learning new words" on TIS, item selection speed, number of errors, rate of word prediction use, satisfaction, cognitive load and perception of speed, a 2 factors repeated-measures analysis of variance was performed with level of lesion as the first factor and activation of word 
prediction software as the second factor (WITHOUT, NOT_ACTIV, ACTIV). A post-hoc method was carried out with a Bonferroni correction.

The level of significance was fixed at $p<0.05$. Data were analyzed using STATISTICA 10 software - StatSoft. Inc software (Tulsa, USA).

\section{Results}

\section{Participants}

Among 90 persons with cervical SCI who fulfilled the inclusion/exclusion, 45 participants with cervical SCI accepted to participate to this study (35 males and 10 females; 39.6 (SD 10) years old; 14.7 (SD 3) years of education). Time since lesion was $10.6 \pm 8$ years. Thirty six participants had used computers for over 10 years, 5 participants between 5 and 10 years, 3 between 1 and 5 years and 1 for less than 1 year.

Lesion level was high (C4 and C5 Asia AIS A or B) for 15 participants (high lesion group) and was between C6 and C8 Asia AIS A or B for 30 participants (low lesion group). In the high lesion group, 13 participants used word processing software regularly (> 3 times per week) and 2 did not ( $\leq 3$ times per month). In the low lesion group (30 participants), all participants used word processing software regularly (> 3 times per week).

Results of the quantitative assessments

Characters per minute (cpm)

The table 1 showed mean number of characters per minute for the three conditions in all participants, participants with low lesions and with high lesions. 
Table 1. Characters per minute - Mean (sd)

\section{Condition}

\section{WITHOUT}

NOT_ACTIV

\section{ACTIV}

All participants

$53.8(37)$

$45.6(22)$

$51.3(23)$

Participants with low

69 (36)

$55.2(19)$

$59.8(21)$

lesions

Participants with high

$23.4(12)$

$26.2(14)$

$34.4(20)$

lesions

There was a significant effect of condition (WITHOUT / NOT_ACTIV / ACTIV) $(F=3.81$;

$p=0.02)$ and lesion level $(F=23.8 ; p<0.001)$ and a significant interaction between condition and lesion level $(F=9.39 ; p<0.001)$.

The Post-Hoc analysis indicated that:

- Participants with a low lesion wrote faster than participants with a high lesion, whatever the condition.

- In participants with low lesions, TIS was significantly higher in the WITHOUT condition than the NOT_ACTIV $(p<0.001)$ and ACTIV $(p=0.02)$ conditions.

- In participants with high lesions, TIS was significantly higher in the ACTIV than the NOT_ACTIV $(p=0.002)$ and WITHOUT $(p<0.001)$ conditions. There was no difference between the NOT_ACTIV and the WITHOUT conditions $(p=0.99)$

\section{Number of Errors}

The table 2 showed mean number of errors for the three conditions in all participants, participants with low lesions and with high lesions. 
Table 2. Number of errors - Mean (sd)

\section{Condition}

WITHOUT

NOT_ACTIV

ACTIV

All participants

$15(10)$

$13.6(8)$

$9.7(5)$

Participants with low

$18.2(9)$

$16.9(6)$

$11.9(5)$

lesions

Participants with high

$8.8(8)$

$6.9(5)$

$5.1(3)$

lesions

There was a significant effect of condition (WITHOUT / NOT_ACTIV / ACTIV) ( $F=9.27$;

$p<0.001)$ and a significant effect of lesion level $(F=26.4 ; p<0.001)$ but no interaction between

the condition and lesion level $(F=1.03 ; p=0.35)$.

The post-Hoc analysis indicated that:

- Participants with low lesions made more errors than participants with high lesions $(p<0.001)$.

- In participants with low lesions, the number of errors was significantly lower in the ACTIV condition than the NOT_ACTIV $(p=0.006)$ and WITHOUT $(p<0.001)$ conditions. There was no difference between the NOT_ACTIV and WITHOUT conditions $(p=0.99)$.

- In participants with high lesions, there was no difference between conditions (ACTIV versus NOT_ACTIV $: p=0.99$, ACTIV versus WITHOUT: $p=0.89$ and NOT_ACTIV versus WITHOUT: $p=0.99)$.

Likewise, it can be noticed that, in participants with low lesions and who had the same levels of errors as participants with high lesions, there was a significant effect of condition (WITHOUT / NOT_ACTIV / ACTIV) $(F=17.7 ; p<0.001)$. The post-Hoc analysis indicated 
that TIS was significantly higher in the WITHOUT condition than the NOT_ACTIV $(p<0.001)$ and ACTIV $(p<0.001)$ conditions.

Item selection speed.

The table 3 showed mean key selection speed for the three conditions in all participants, participants with low lesions and with high lesions.

Table 3. Key selection speed (keys per minute) - Mean (sd)

\section{Condition}

\section{WITHOUT}

\section{NOT_ACTIV}

$52.2(25)$

$63(22)$

$67.4(22)$

lesions

Participants with high

$25.4(13)$

$30(16)$

$39.8(23)$

lesions

There was a significant effect of condition (WITHOUT / NOT_ACTIV / ACTIV) ( $F=3.38$;

$p=0.03)$ and lesion level $(F=24.2 ; p<0.001)$ as well as a significant interaction between

condition and lesion level $(F=12.7 ; p<0.001)$.

The post-Hoc analysis indicated that:

-Participants with low lesions selected keys more quickly than participants with high lesions.

-For participants with low lesions, key selection speed in the WITHOUT condition was

significantly faster than in the NOT_ACTIV $(p<0.001)$ and ACTIV $(p=0.02)$ conditions. 
-For participants with high lesions, key selection speed was significantly faster in the ACTIV condition than in the NOT_ACTIV $(p=0.004)$ and WITHOUT $(p<0.001)$ conditions. There was no difference between the NOT_ACTIV and WITHOUT conditions $(p=0.99)$.

\section{Rate of Word prediction use}

The table 4 showed mean rate of word prediction use for the three conditions in all participants, participants with low lesions and with high lesions.

Table 4. Rate of Word prediction use - Mean (sd)

\section{Condition}

\section{WITHOUT}

NOT_ACTIV

\section{ACTIV}

All participants

N/A

29.8 (17)

$51.7(25)$

Participants with low

N/A

$31.9(17)$

$53(18)$

lesions

Participants with high

N/A

$25.8(17)$

$49(36)$

lesions

N/A: Not Applicable

There was a significant effect of condition (NOT_ACTIV / ACTIV) $(F=37.5 ; p<0.001)$ but no significant effect of lesion level $(F=0.7 ; p=0.39)$ and no interaction between condition and lesion level $(F=0.09 ; p=0.76)$

The post-hoc analysis indicated that:

- The rate of word prediction use was significantly higher in the ACTIV than NOT_ACTIV condition in participants with low $(p<0.001)$ and high lesions $(p=0.01)$. 
Results of the qualitative assessments

Subjective measures (fatigue, perception of speed, cognitive load and satisfaction)

The table 5 showed results of qualitative outcomes for the three conditions in all participants, participants with either low lesions or with high lesions. 
Table 5. Results of qualitative outcome - Mean (sd)

\section{Qualitative Outcomes}

\section{Condition}

WITHOUT

NOT_ACTIV

ACTIV

\begin{tabular}{|c|c|c|c|c|}
\hline Fatigue & All participants & $1.4(1.7)$ & $1(1.5)$ & $0.7(1.3)$ \\
\hline \multirow[t]{2}{*}{ (difference between the before and after task) } & Participants with low lesions & $1.6(2)$ & $1(1.5)$ & $0.7(1.3)$ \\
\hline & Participants with high lesions & $0.8(0.8)$ & $1(1.5)$ & $0.8(1.4)$ \\
\hline \multirow[t]{3}{*}{ Sensation of speed } & All participants & $5(3)$ & $5(2)$ & $6.3(1.9)$ \\
\hline & Participants with low lesions & $6(1.5)$ & $5.6(1.9)$ & $7.1(1.7)$ \\
\hline & Participants with high lesions & $4(1)$ & $3.3(2.3)$ & $5.7(2.2)$ \\
\hline \multirow[t]{3}{*}{ Cognitive load } & All participants & $5(2)$ & $6.2(1.8)$ & $5.4(2)$ \\
\hline & Participants with low lesions & $5(2)$ & $6.4(1.4)$ & $5.4(1.7)$ \\
\hline & Participants with high lesions & $5(2)$ & $5.7(2.1)$ & $5.3(2.5)$ \\
\hline \multirow[t]{3}{*}{ Satisfaction } & All participants & $3(2)$ & $3.2(1)$ & $3.9(1)$ \\
\hline & Participants with low lesions & $3(2)$ & $3(0.9)$ & $3.8(1)$ \\
\hline & Participants with high lesions & $3(2)$ & $3.8(0.9)$ & $4(0.9)$ \\
\hline
\end{tabular}


Regarding fatigue, there was no significant effect of condition (WITHOUT / NO_ACTIV / ACTIV) $(F=1.01 ; p=0.36)$, lesion level $(F=0.34 ; p=0.56)$ and no interaction between condition and lesion level $(F=1.62 ; p=0.20)$.

Regarding perception of speed, there was a significant effect of condition (WITHOUT / NO_ACTIV / ACTIV) $(F=26.8 ; p<0.001)$ but no effect of lesion level $(F=2 ; p=0.16)$; there was no interaction between condition and lesion level $(F=1.2 ; p=0.27)$.

The post-hoc analysis indicated that:

-Participants with low lesions perceived that they inputted text significantly faster in the ACTIV than the WITHOUT $(p<0.001)$ or NOT_ACTIV $(p<0.001)$ conditions. There was no difference between the NOT_ACTIV and WITHOUT conditions $(p=0.99)$.

-For participants with high lesions, there was no difference between the WITHOUT and NOT_ACTIV $(p=0.99)$ or ACTIV $(p=0.09)$ conditions.

Regarding cognitive load, there was a significant effect of condition (WITHOUT / NO_ACTIV / ACTIV $)(F=6.45 ; p=0.01)$ but no effect of lesion level $(F=0.45 ; p=0.5)$ and no interaction between condition and lesion level $(F=1.16 ; p=0.28)$.

The post-hoc analysis indicated that:

-For participants with low lesions, cognitive load was significantly higher in the NOT_ACTIV than WITHOUT condition $(p=0.01)$. For the high lesion group, there were no differences between the WITHOUT, NOT_ACTIV $(p=0.99)$ and ACTIV ( $p=0.99)$ conditions. Regarding satisfaction with WPS, there was a significant effect of condition (WITHOUT / NO_ACTIV / ACTIV $)(F=17.5 ; p<0.001)$ but no effect of lesion level $(F=3.5 ; p=0.06)$. There was also a significant interaction between condition and lesion level $(F=6.8 ; p=0.01)$. The post-hoc analysis indicated that: -Participants with low lesions were more satisfied after the completion of a copying task with the ACTIV condition than the NOT_ACTIV condition $(p<0.001)$. 
-For the high lesion group, there was no difference between the NOT_ACTIV $(p=0.99)$ and $\operatorname{ACTIV}(p=0.99)$ conditions.

\section{Discussion}

The hypothesis of this study was that the activation of the frequency of use and learning new words parameters would increase TIS in a homogenous population of persons with cervical SCI AIS A or B and therefore that in the ACTIV condition participants would input text faster than in the NOT_ACTIV or WITHOUT conditions.

However, the results showed that the effects differed according to the level of the lesion: i) for participants with low lesions, TIS was significantly higher when WPS was not used ; ii) for participants with high lesions, text input was significantly faster when using WPS with the frequency of use and automatic learning parameters activated. The hypothesis was thus confirmed only in participants with high lesions.

The increase in TIS found in the high lesion group with use of WPS in the ACTIV condition is not in accordance with the results of a previous study which showed that the use of WPS decreases TIS [19]. However, two participants with high SCI and four participants with low lesions were included in that study and the results were not separated according to the level of the lesion. The results of the present study, obtained in a larger sample of subjects, suggest that these WPS parameters can be useful for persons with high spinal cervical injuries. This was further confirmed by the high rate of word prediction use in the ACTIV condition in this group. It is interesting that despite the increase of around 30\% in TIS and the doubling of the rate of use of word prediction in the ACTIV compared with the NOT-ACTIV condition, the participants did not perceive the increase in speed. It is possible that this increase is not perceived because TIS remained very low. Therapists therefore need to be aware of the objective effects of the use of certain software and parameters and must consider the 
perception of users with caution when prescribing and setting up communication devices for patients, particularly since activation of these parameters did not increase cognitive load. The results also showed that use of WPS did not decrease the number of errors. This may be because the low TIS indirectly limited the number of errors or because the location of onscreen keyboard restricted visual exploration.

In contrast with the high lesion group, activation of the frequency of use and automatic learning parameters did not improve TIS in the low lesion group. However, rate of word prediction use was higher when these parameters were activated. More surprisingly, the results of this study, conducted in a relatively large sample, suggested that when the level of the lesion was at or below C6 Asia AIS A and B [20] TIS was faster without WPS. However, use of WPS did appear to provide some advantages. For example, the number of errors was reduced when the frequency of use and automatic learning parameters were activated. This reduction may result in the sensation of wasting less time to correct errors, even if this was not confirmed objectively. Moreover, regarding the number of errors, this result is in accordance with the data in the literature. Antoine et al. (2007) showed in ten participants with cerebral palsy that WPS did not necessarily increase TIS but reduced the number of errors and fatigue [21]. However, regarding fatigue, the results of the present study do not confirm their results since there was no effect of WPS on fatigue in either group. This difference might be related to the fact that persons with cervical SCI do not experience fatigue to the same extent as persons with brain injury [22]. Indeed, persons with cerebral palsy people frequently have a certain degree of cognitive impairment. Thus typing requires more attention and generates more fatigue. Another factor may be that since all the participants of the present study had been regular computer users since their accidents, they may have built up endurance to text input. 
An important result which arose from this study is that the activation of the frequency of use and automatic learning parameters influenced either TIS or the number of errors depending on the level of injury. This suggests that inconstancies in the literature relating to the effect of WPS on TIS may relate to a lack of standardization of the activation of associated parameters across studies [23] [17] [5] as well as the inclusion of subjects with different levels of cervical injury. In order to fully optimize text input in persons with cervical SCI, it is thus fundamental to study the effects of different software in more detail, mainly the effect of the different parameters within each software. It is also essential to understand the settings prescribed by health professionals and the criteria on which they base their choices.

It was also surprising to find that the participants did not perceive the objective improvements in TIS with the use of WPS. It is therefore important that therapists should carry out objective measures to aid patients in the choice of software and communication devices. This study involved a single typing session and the WPS was new to all participants. It is possible that a period of training or longer-term use of WPS would have allowed improvements to be consolidated and participants to become aware of the benefits of the software and parameters.

\section{Limitations}

The difference in the size of the two groups (high and low cervical SCI) may have limited the interpretation of results. Similarly differences in the frequency of use of word processing between subjects could have increased the variability of TIS.

\section{Conclusion}


The aims of this study were to determine if the activation of the frequency of use and automatic learning parameters of WPS affected TIS in persons with cervical SCI. The results showed that the effects differed according to the level of the lesion. In participants with lesions at or below $\mathrm{C} 6$, the activation of these parameters decreased the number of errors but did not increase TIS. In contrast, in participants with lesions above C6, the activation of these parameters increased TIS but did not decrease the number of errors. These results demonstrate the importance of appropriately setting WPS parameters both when prescribing systems for patients and in future studies. Finally, participants did not perceive improvements associated with the WPS. It would be interesting to evaluate the effects of a training period with WPS to evaluate if subjects become aware of changes and if further improvements occur. 


\section{ACKNOWLEDGMENTS}

We sincerely thank all the participants of this study.

\section{DECLARATION OF INTEREST}

This research is supported by the Paul Bennetot Fondation (Matmut Group Foundation)

\section{CONFLICT OF INTEREST STATEMENT}

None of the authors have any declaration of interest to report regarding this study. 


\section{References}

[1] Bigot P, Croutte E. La diffusion des technologies de 1' information et de la communication dans la société. Rapp réalisé à la demande du Cons Général des Technol l'Information (Ministère l'Economie, des Financ l'Emploi) l'Autorité Régulation des Commun Electron des Postes Cent Rech pour l'Etude l'O. 2013:220.

[2] Laffont I, Biard N, Bouteille J, Pouplin S, Guillon B, Bernuz B, Rech C. Tétraplégie: solutions technologiques de compensation des incapacités découlant de l'atteinte des membres supérieurs. La Lett médecine Phys réadaptation [Internet]. 2008 September 27 [cited 2012 December 6];24:113-121. Available from: http://www.springerlink.com/index/10.1007/s11659-008-0106-y

[3] Betke M, Gips J, Fleming P. The camera mouse: visual tracking of body features to provide computer access for people with severe disabilities. IEEE Trans Neural Syst Rehabil Eng [Internet]. 2002 March;10:1-10. Available from: http://www.ncbi.nlm.nih.gov/pubmed/12173734

[4] Dalton JR, Peterson CQ. The use of voice recognition as a control interface for word processing. Occup Ther Heal care [Internet]. 1997 January;11:75-81. Available from: http://www.ncbi.nlm.nih.gov/pubmed/23931591

[5] Koester HH, Levine SP. Effect of a Word Prediction Feature on User Performance. Augment Altern Commun [Internet]. 1996;12:155-168. Available from: http://informahealthcare.com/doi/abs/10.1080/07434619612331277608

[6] Lau C, Leary SO. Comparison of computer interface devices for persons with severe physical disabilities. Am J Occup Ther. 1993;47.

[7] DeVries RC, Deitz J, Anson D. A Comparison of Two Computer Access Systems for Functional Text Entry. Am J Occup Ther. 1998;52:656-665.

[8] Le Pévédic B. Prédiction Morphosyntaxique Évolutive HandiAS. Thesis. 1997.

[9] Turpin G, Armstrong J, Frost P, Fine B, Ward C, Pinnington L. Evaluation of alternative computer input devices used by people with disabilities. J Med Eng Technol [Internet]. 2005;29:119-29. Available from: http://www.ncbi.nlm.nih.gov/pubmed/16019881

[10] MacKenzie IS, Zhang SX. The design and evaluation of a high-performance soft keyboard. Proc SIGCHI Conf Hum factors Comput Syst CHI is limit - CHI '99 
[Internet]. 1999:25-31. Available from:

http://portal.acm.org/citation.cfm?doid=302979.302983

[11] Raynal M, Vigouroux N. Genetic algorithm to generate optimized soft keyboard. CHI '05 Ext Abstr Hum factors Comput Syst - CHI '05 [Internet]. 2005:1729. Available from: http://portal.acm.org/citation.cfm?doid=1056808.1057008

[12] Kushler C. AAC using a reduced keyboard. Proc CSUN Conf Technol Pers with Disabil CSUN'98 Calif State Univ Nortridge CA. 2001.

[13] Harbusch. An evaluation an evaluation study of two button scanning with ambiguous keyboards. In: 7th Conference for the Advancement of Assistive Technology in Europe, AATE'2003. Dublin, Ireland. 2003.

[14] Dalton JR, Peterson CQ. The use of voice recognition as a control interface for word processing. Occup Ther Heal care. 1997 January;11:75-81.

[15]. Wandmacher T, Antoine J, Poirier F, Départe J-P. Sibylle, An Assistive Communication System Adapting to the Context and Its User. ACM Trans Access Comput. 2008.

[16] Pouplin S, Roche N, Hugeron C, Isabelle V, Bensmail D. Recommendations and settings of word prediction software by health-related professionals for people with spinal cord injury : a prospective observational study. Eur J Phys Rehabil Med. 2015:article in press.

[17] Koester HH, Levine S. Keystroke-level models for user performance with word prediction. Augment Altern Commun [Internet]. 1997;13:239-257. Available from: http://informahealthcare.com/doi/abs/10.1080/07434619712331278068

[18]. Fraval Lye M, Boutard C. Textzados. Ortho Edit. Isbergues; 2004.

[19] Koester HH. Effect of system configuration on user performance with word prediction result for users with disabilities. In: Proceedings of the RESNA 2000: June 28 - July 2. 2000.

[20] Guttmann L. Spinal Cord Injuries, Comprehensive Management and Research. 1ere éditi. Blackwell Science Ltd; 1973.

[21] Antoine J, Maurel D. Aide à la communication pour personnes handicapées et prédiction de texte Problématique, état des lieux et retour sur trente ans. TAL. 2007;48:9-46. 
[22] Choi-Kwon S., Jong S. K. Poststroke fatigue: an emerging, critical issue in stroke medicine. International Journal of Stroke, Vol 6, August 2011, 328-336

[23] Anson D, Moist P, Przywara M, Wells H, Saylor H, Maxime H. The effects of word completion and word prediction on typing rates using on-screen keyboards. Assist Technol [Internet]. 2006 January; 18:146-54. Available from:

http://www.ncbi.nlm.nih.gov/pubmed/17236473 
TABLES

Table 1. Characters per minute - Mean (sd)

\begin{tabular}{cccc} 
& \multicolumn{3}{c}{ Condition } \\
\hline All participants & $53.8(37)$ & NOT_ACTIV & ACTIV \\
Participants with low & $69(36)$ & $45.6(22)$ & $51.3(23)$ \\
lesions & & $55.2(19)$ & $59.8(21)$ \\
Participants with high & $23.4(12)$ & & $34.4(20)$ \\
lesions & & & \\
\hline
\end{tabular}


Table 2. Number of errors - Mean (sd)

\begin{tabular}{cccc} 
& \multicolumn{3}{c}{ Condition } \\
\hline & WITHOUT & NOT_ACTIV & ACTIV \\
All participants & $15(10)$ & $13.6(8)$ & $9.7(5)$ \\
Participants with low & $18.2(9)$ & $16.9(6)$ & $11.9(5)$ \\
lesions & & & $5.1(3)$ \\
Participants with high & $8.8(8)$ & $6.9(5)$ & \\
\hline lesions & & & \\
\hline
\end{tabular}


Table 3. Key selection speed (keys per minute) - Mean (sd)

\begin{tabular}{cccc} 
& \multicolumn{3}{c}{ Condition } \\
\hline All participants & $69.9(45)$ & NOT_ACTIV & ACTIV \\
Participants with low & $81.8(43)$ & $52.2(25)$ & $58.2(25)$ \\
lesions & & & $67.4(22)$ \\
Participants with high & $25.4(13)$ & $30(16)$ & $39.8(23)$ \\
lesions & & & \\
\hline
\end{tabular}


Table 4. Rate of Word prediction use - Mean (sd)

\begin{tabular}{cccc} 
& \multicolumn{3}{c}{ Condition } \\
\hline & WITHOUT & NOT_ACTIV & ACTIV \\
All participants & N/A & $29.8(17)$ & $51.7(25)$ \\
Participants with low & N/A & $31.9(17)$ & $53(18)$ \\
lesions & & & \\
Participants with high & N/A & $25.8(17)$ & \\
lesions & & & \\
\hline
\end{tabular}

N/A: Not Applicable 
Table 5. Results of qualitative outcome - Mean (sd)

\section{Qualitative Outcomes}

\section{Condition}

WITHOUT

NOT_ACTIV

ACTIV

\begin{tabular}{|c|c|c|c|c|}
\hline Fatigue & All participants & $1.4(1.7)$ & $1(1.5)$ & $0.7(1.3)$ \\
\hline \multirow[t]{2}{*}{ (difference between the before and after task) } & Participants with low lesions & $1.6(2)$ & $1(1.5)$ & $0.7(1.3)$ \\
\hline & Participants with high lesions & $0.8(0.8)$ & $1(1.5)$ & $0.8(1.4)$ \\
\hline \multirow[t]{3}{*}{ Sensation of speed } & All participants & $5(3)$ & $5(2)$ & $6.3(1.9)$ \\
\hline & Participants with low lesions & $6(1.5)$ & $5.6(1.9)$ & $7.1(1.7)$ \\
\hline & Participants with high lesions & $4(1)$ & $3.3(2.3)$ & $5.7(2.2)$ \\
\hline \multirow[t]{3}{*}{ Cognitive load } & All participants & $5(2)$ & $6.2(1.8)$ & $5.4(2)$ \\
\hline & Participants with low lesions & $5(2)$ & $6.4(1.4)$ & $5.4(1.7)$ \\
\hline & Participants with high lesions & $5(2)$ & $5.7(2.1)$ & $5.3(2.5)$ \\
\hline \multirow[t]{3}{*}{ Satisfaction } & All participants & $3(2)$ & $3.2(1)$ & $3.9(1)$ \\
\hline & Participants with low lesions & $3(2)$ & $3(0.9)$ & $3.8(1)$ \\
\hline & Participants with high lesions & $3(2)$ & $3.8(0.9)$ & $4(0.9)$ \\
\hline
\end{tabular}

